\title{
Conjuguer développement professionnel continu et amélioration de la qualité
}

\author{
Wendy Levinson MD, Brian M. Wong MD \\ Citation : CMAJ 2021 May 3;193:E647-8. doi : 10.1503/cmaj.202797-f \\ Voir la version anglaise de l'article ici : www.cmaj.ca/lookup/doi/10.1503/cmaj.202797
}

$\mathbf{L}$ es activités classiques de développement professionnel continu (DPC) permettent aux médecins d'améliorer leurs connaissances, mais ratent en général la cible pour ce qui est de changer les pratiques cliniques ou d'améliorer les résultats pour les patients'. Récemment, L'Avenir de l'éducation médicale au Canada a recommandé l'adoption d'une nouvelle approche de DPC en appui à l'amélioration de la pratique et de la santé au pays ${ }^{2}$. Cette approche implique un passage de l'apprentissage passif, axé sur les cours magistraux, à un apprentissage plus actif faisant intervenir les médecins dans le processus d'amélioration de la qualité (AQ). On parle entre autres ici de l'évaluation des indicateurs, de la comparaison de ces derniers aux indices de référence, et de l'élaboration de plans d'amélioration mesurable, selon les besoins. Nombre de médecins n'ont pas encore intégré l'AQ à leur pratique en raison d'obstacles tels que le manque d'intérêt, de temps, de mesures incitatives ou de connaissances des méthodes d'AQ, et l'absence de données cliniques pertinentes ${ }^{3-6}$. Le présent article porte sur la possibilité de changer les mentalités compte tenu des incitatifs découlant de l'intégration des activités d'AQ à la certification, aux exigences professionnelles et aux systèmes.

Le programme de maintien du certificat du Collège royal des médecins et chirurgiens du Canada (CRMCC) requiert l'évaluation des pratiques d'après les commentaires de pairs ou une comparaison avec les indices de référence, un procédé associé à des ajustements dans les habitudes professionnelles et à de meilleurs résultats pour les patients ${ }^{7}$. Les activités concernant l'évaluation s'inscrivent dans la « section 3 ». L'incitatif du CRMCC est d'offrir aux spécialistes 3 crédits au programme par heure consacrée à une telle activité. De nombreux spécialistes prennent probablement déjà part à des projets d'AQ donnant accès à des crédits de la section 3. Environ 150 hôpitaux canadiens participent à un projet national récemment lancé pour réduire les transfusions inappropriées de culots globulaires ${ }^{8}$. Celui-ci mobilise divers spécialistes (p. ex., en chirurgie, médecine interne, hématologie, anesthésiologie et médecine transfusionnelle) qui font un suivi des taux de transfusions pour les comparer aux indices de référence nationaux sur l'utilisation appropriée et ajuster les pratiques de leur hôpital. Cette démarche d'AQ permet l'obtention de crédits à la section 3.

\section{POINTS CLÉS}

- Le développement professionnel continu intégrant l'amélioration de la qualité est probablement plus efficace que les méthodes d'apprentissage passif lorsqu'il est question de changer les pratiques cliniques et d'améliorer les résultats pour les patients.

- Les médecins effectuent peut-être déjà des activités d'amélioration de la qualité qui répondent aux nouvelles exigences réglementaires.

- Les organisations de soins de santé, comme les hôpitaux et les cabinets de médecine familiale, doivent de plus en plus rendre des comptes sur leurs indicateurs de qualité, et le leadership des médecins est essentiel à cette démarche.

Le Collège des médecins de famille du Canada (CMFC) exige quant à lui la participation de ses membres au programme de DPC Mainpro+ ${ }^{+}$. II élabore également un «Plan d'apprentissage professionnel » pour encourager les médecins de famille à évaluer leur rendement, à réfléchir aux moyens d'améliorer leurs pratiques et à apporter les changements nécessaires. Les médecins de famille peuvent notamment procéder à une vérification d'aspects de leurs pratiques, comme la prescription d'antibiotiques ou d'opioïdes, en vue d'améliorer leurs soins aux patients. Ils compareront idéalement leurs pratiques aux pratiques exemplaires ou à celles de leurs pairs, ce qui n'est pas toujours possible étant donné le manque de données disponibles.

Les autorités de réglementation provinciales empruntent la même voie en remplaçant les vérifications aléatoires effectuées par les pairs, qui n'ont que peu d'effets sur les changements de pratiques, par une participation obligatoire des médecins aux initiatives d'AQ de leur établissement. Par exemple, l'Ordre des médecins et chirurgiens de l'Ontario (OMCO) a remplacé son programme de vérifications existant par un nouveau qui permet aux médecins de faire reconnaître leurs contributions aux initiatives $d^{\prime} A Q$ de leur établissement ${ }^{10}$. Les hôpitaux soumettent à l'Ordre des données sur les activités d'amélioration et lui certifient que tel ou tel médecin y a participé. Un programme semblable a été mis sur pied pour les médecins exerçant en milieu communautaire. 
Les autorités de réglementation d'autres provinces conçoivent actuellement des programmes d'évaluation comparables dont dépendra le permis d'exercice.

Les nouvelles ententes de financement entre les gouvernements provinciaux et les organisations de soins de santé se rattachent aussi de plus en plus à des indices de référence de la qualité. En Alberta, le financement des réseaux de soins primaires dépend de l'évaluation des indicateurs de la qualité en soins primaires, comme le temps d'attente pour un rendez-vous, le respect des normes de pratique professionnelle en matière de dépistage et de prévention, et d'autres mesures ciblées (p. ex., prescription d'opioïdes) ${ }^{11}$. Certaines provinces obligent les hôpitaux à faire rapport des indicateurs de qualité, comme les résultats déclarés par le patient après une opération à la hanche ou au genou ${ }^{12}$. Le succès de ces approches systémiques repose sur la mobilisation de médecins qui guident les démarches et établissent des indicateurs de rendement fondés sur données probantes, pertinents sur le plan clinique et jugés acceptables par leurs pairs.

La participation accrue des médecins à l'AQ s'accompagne de questions et d'enjeux non résolus. L'amélioration de la qualité est

Encadré 1 : Activités d'amélioration de la qualité pouvant s'inscrire dans le développement professionnel continu

Médecins de famille

Vérification des dossiers pour un sujet donné et réflexion sur les possibilités d'amélioration, en solo ou avec des collègues

Autoévaluation à l'aide d'un outil en ligne (p. ex., eCoach de l'UBC)

Réflexion sur les rapports de pratique générés à partir des DME et mettant en parallèle la qualité des soins prodigués dans le cabinet et dans d'autres cabinets comparables

Revue des résultats de sondages sur l'expérience des patients tenus par le cabinet

Utilisation de trousses d'outils pour l'AQ en soins primaires (p. ex., outils de Choisir avec soin pour la déprescription de médicaments)

Participation à un programme de DPC axé sur les approches d'AQ

Spécialistes exerçant en milieu hospitalier

Étude des données sollicitées (ou fournies) par les services d'aide à la prise de décisions des hôpitaux pour établir les améliorations possibles

Participation aux examens réguliers de la morbidité et de la mortalité et à l'évaluation d'un incident ayant mis en jeu la sécurité d'un patient

Revue des rapports sur le rendement qui comparent les résultats des patients hospitalisés à ceux d'autres hôpitaux (p. ex., programme NSQIP de l'American College of Surgeons)

Réflexion sur les résultats des rapports ciblant l'expérience des patients d'une unité médicale

Contribution à une initiative d'AQ de l'hôpital en tant que membre d'une équipe multidisciplinaire

Participation à un programme de DPC axé sur les approches d'AQ

Remarque : $\mathrm{AQ}$ = amélioration de la qualité, $\mathrm{DME}$ = dossier médical électronique, $\mathrm{DPC}=$ développement professionnel continu, NSQIP = programme national d'amélioration de la qualité des soins chirurgicaux, UBC = Université de la Colombie-Britannique. souvent multidisciplinaire; or, les organismes d'agrément se bornent à évaluer le rendement individuel des médecins. Ces derniers pourraient avoir besoin d'une formation et d'une aide complémentaires pour intégrer l'AQ à leurs pratiques. Les administrateurs des hôpitaux sont bien placés pour leur fournir les données et le soutien essentiels à l'amélioration, mais cet appui est souvent déficient en milieu de soins primaires. Les données servant à la mesure du rendement seraient idéalement générées automatiquement par le système de dossiers médicaux électroniques, mais en l'absence d'un tel système, la simple vérification des dossiers pourrait faire l'affaire. Par ailleurs, les données permettant la comparaison entre les médecins et leurs pairs ou les indices de référence ne sont pas toujours disponibles.

En conjuguant $\mathrm{DPC}$ et $\mathrm{AQ}$, on ouvre la porte à plusieurs activités bénéfiques pour les médecins, tant en milieu ambulatoire qu'en milieu hospitalier (encadré 1 ). Ces activités leur permettront à la fois de mieux soigner leurs patients et d'obtenir des crédits obligatoires pour répondre aux exigences. Malgré les défis à relever, la vision commune des organismes d'accréditation et de réglementation en matière de DPC et d'AQ favorise la participation concrète des médecins aux activités d'AQ.

\section{Références}

1. Davis D, O'Brien MA, Freemantle N, et al. Impact of formal continuing medical education: do conferences, workshops, rounds, and other traditional continuing education activities change physician behavior or health care outcomes? JAMA 1999;282:867-74.

2. Campbell C, Sisler, J, Future of Medical Education in Canada CPD Steering Committee. Summary report of the Future of Medical Education in Canada Continuing Professional Development (FMEC CPD) Project. Coalition for Physician Learning and Practice Improvement; 2019. Accessible ici : www.afmc.ca/sites/ default/files/pdf/2019-FMEC-MD_EN.pdf (consulté le 16 févr. 2021).

3. Berwick DM. A primer on leading the improvement of systems. BMJ 1996;312:619-22.

4. Kwok ES, Perry J, Mondoux S, et al. An environmental scan of quality improvement and patient safety activities in emergency medicine in Canada. CJEM 2019;21:535-41.

5. Eva KW, Regehr G. Effective feedback for maintenance of competence: from data delivery to trusting dialogues. CMAJ 2013;185:463-4.

6. Dave Davis DA, McMahon GT. Translating evidence into practice: lessons for CPD. Med Teach 2018;40:892-5.

7. About the MOC program. Ottawa: Royal College of Physicians and Surgeons of Canada. Accessible ici : https://www.royalcollege.ca/rcsite/cpd/moc-program/ about-moc-program-e (consulté le 16 févr. 2021).

8. Using Blood Wisely. Choosing Wisely Canada/Ottawa: Canadian Blood Services. Accessible ici : https://usingbloodwisely.ca (consulté le 17 févr. 2021).

9. Understanding Mainpro+ certification. Mississauga (ON): College of Family Physicians of Canada; 2020. Accessible ici : https://portal.cfpc.ca/resourcesdocs/ uploadedFiles/CPD/Mainpro_-_Maintenance_of_Proficiency/CPD_Providers_and _Planners/Mainpro-Certification-Standards.pdf (consulté le 11 déc. 2020).

10. QI partnership for hospitals. Toronto: College of Physicians and Surgeons of Ontario. Accessible ici : https://www.cpso.on.ca/Physicians/Your-Practice/ Quality-Improvement-Program/QI-Partnership (consulté le 22 févr. 2021).

11. A case study evaluation of Crowfoot Village Family Practice and the Taber Clinic. Calgary: Health Quality Council of Alberta; 2019. Accessible ici : https://hqca.ca/wp -content/uploads/2019/12/HQCA-Crowfoot_Taber-Case-Study-Evaluation-2019 .pdf (consulté le 11 déc. 2020).

12. White paper: enhancing the use of patient-reported outcome measures (PROMs) in the healthcare system in Alberta. Edmonton: Alberta PROMs and EQ-5D Research and Support Unit (APERSU) School of Public Health University of Alberta; 2020. Accessible ici : https://apersu.ca/wp-content/uploads/2020 /09/APERSU-PROMs-White-Paper.pdf (consulté le 14 déc. 2020). 
Intérêts concurrents : Aucun déclaré.

Cet article a été révisé par des pairs.

Affiliations : Faculté de médecine Temerty (Levinson, Wong), Université de Toronto; Centre des sciences de la santé Sunnybrook (Wong), Toronto, Ont.

Contributeurs : Wendy Levinson et Brian Wong ont conceptualisé l'article. Wendy Levinson en a rédigé la première version, et Brian Wong a révisé les versions subséquentes. Les deux auteurs ont donné leur approbation finale pour la version soumise pour publication et assument l'entière responsabilité de tous les aspects du travail.

Propriété intellectuelle du contenu : Il s'agit d'un article en libre accès distribué conformément aux modalités de la licence Creative Commons Attributions (CC BY-NC-ND 4.0), qui permet l'utilisation, la diffusion et la reproduction dans tout médium à la condition que la publication originale soit adéquatement citée, que l'utilisation se fasse à des fins non commerciales (c.-à-d., recherche ou éducation) et qu'aucune modification ni adaptation n'y soit apportée. Voir : https://creativecommons.org/licenses/by-nc-nd/4.0/deed.fr.

Remerciements : Les auteurs souhaitent remercier Karen Born pour son aide à la révision du manuscrit.

Correspondance : Wendy Levinson, wendy.levinson@utoronto.ca 